\title{
EL PAPEL DE LAS VÍAS SECUNDARIAS Y LOS CAMINOS VECINALES EN EL DESARROLLO DE COLOMBIA
}

\section{The role of secondary and tertiary roads in the development of Colombia}

\author{
Germán Ospina Ovalle \\ Ingeniero Civil, especialista en Gestión Vial. Contacto: go.geosov@gmail.com
}

\section{Resumen}

Las vías Secundarias y Terciarias juegan un papel importante en la integración nacional, regional y local, y además facilitan el acceso a la vida nacional de comunidades remotas y aisladas. Estas vías en su conjunto, representan cerca del $92 \%$ del total de la malla vial nacional. Este artículo señala algunos puntos que deben considerarse durante la planeación y ejecución de la infraestructura vial secundaria y terciaria, importante si el Gobierno Nacional quiere materializar el posconflicto en el país rural.

Palabras clave: Vías secundarias, vías terciarias, infraestructura vial, posconflicto, inversión estatal.

\section{Abstract}

Secondary and tertiary roads play an important role in national, regional and local integration, and also facilitate access to the national life of remote and isolated communities. These pathways as a whole, represent about $92 \%$ of total national road network. This article points out some points that should be considered during the planning and execution of secondary and tertiary, important road infrastructure if the Government wants to materialize post-conflict in rural country.

Keywords: Secondary roads, tertiary roads, road infrastructure, postconflict, state investment.

\section{Antecedentes}

En Colombia la gestión vial está a cargo de la nación, los departamentos y los municipios. Cada uno de estos niveles administrativos tienea su cargo una malla vial que va descendiendo en calidad y categoría en la medida que se pasa del primer nivel al tercero, pero que en longitud se incrementa en sentido inverso (sin embargo, no es raro encontrar que algunas carreteras nacionales tienen menores especificaciones geométricas que algunas carreteras departamentales y, a su vez, que algunos caminos vecinales tengan mejores especificaciones que algunas carreteras departamentales).

Actualmente se estima que el Sistema Nacional de Carreteras[1] tiene una longitud de $215.988 \mathrm{~km}$, de los cuales $17.382(8 \%)$ hacen parte de la llamada Red de Primer Orden, gestionada por el Instituto Nacional de Vías (INVÍAS) y la Agencia Nacional de Infraestructura (ANI). La Red Vial de Segundo Orden suma 44.399 km $(21 \%)$ y la Red de Tercer Orden, o Veredal, cuenta con $154.207 \mathrm{~km}(71 \%)$-incluidos cerca de $12.500 \mathrm{~km}$ de 
caminos privados-, los cuales están bajo la gestión de los departamentos, los distritos, los municipios e INVÍAS.

La Red Primaria está constituida por las carreteras que unen las principales ciudades entre sí, con los puertos marítimos, fronteras terrestres y otros nodos de intercambio modal. Esta red es básica para la integración y competitividad del país (pues une a las áreas de producción con las de consumo) y, en su mayor parte, está constituida por vías de doble calzada y carreteras bidireccionales pavimentadas y con especificaciones geométricas adecuadas. En esta red se tienen los mayores movimientos de pasajeros y carga en el país, particularmente en los corredores de comercio exterior. El planeamiento de esta red está definido por el Plan Maestro de Transporte Intermodal[2].

Por otra parte está la Red Secundaria, compuesta por carreteras de calzada bidireccional, con o sin pavimento. Durante largo tiempo hubo desconocimiento sobre las características de esta red, pero gracias a la realización del Plan Vial Regional (PVR), adelantado por el Ministerio de Transporte (MT), actualmente se tiene una idea más clara sobre su longitud y estado. Se sabe, por ejemplo, que cerca de $8.000 \mathrm{~km}$ están pavimentados y que los volúmenes de tránsito son, por lo general, inferiores a 500 vehículos por día.

En el año 1961 las carreteras departamentales tenían una longitud de $14.851 \mathrm{~km}$ y, de acuerdo con varios estudios realizados en esa época[3], su estado era precario, tanto en lo que se refiere a sus especificaciones geométricas, como al estado de la superficie.

Esta red fue creciendo, tanto por la adición de nuevos tramos, como por la transferencia de algunas vías de la Nación a los departamentos y el compromiso que existía en el sentido de que éstos debían encargarse del mantenimiento de las carreteras construidas por el Fondo Nacional de Caminos Vecinales (FNCV), el Instituto Colombiano de Reforma Agraria (INCORA), el programa de Desarrollo Rural Integrado (DRI) y otras entidades, aunque en realidad pocas veces lo hicieron.

Con la expedición de la Ley 105 de 1993, se estableció que las vías primarias deberían pertenecer a la nación y aquellas de carácter secundario deberían ser responsabilidad de los departamentos. Estos, a su

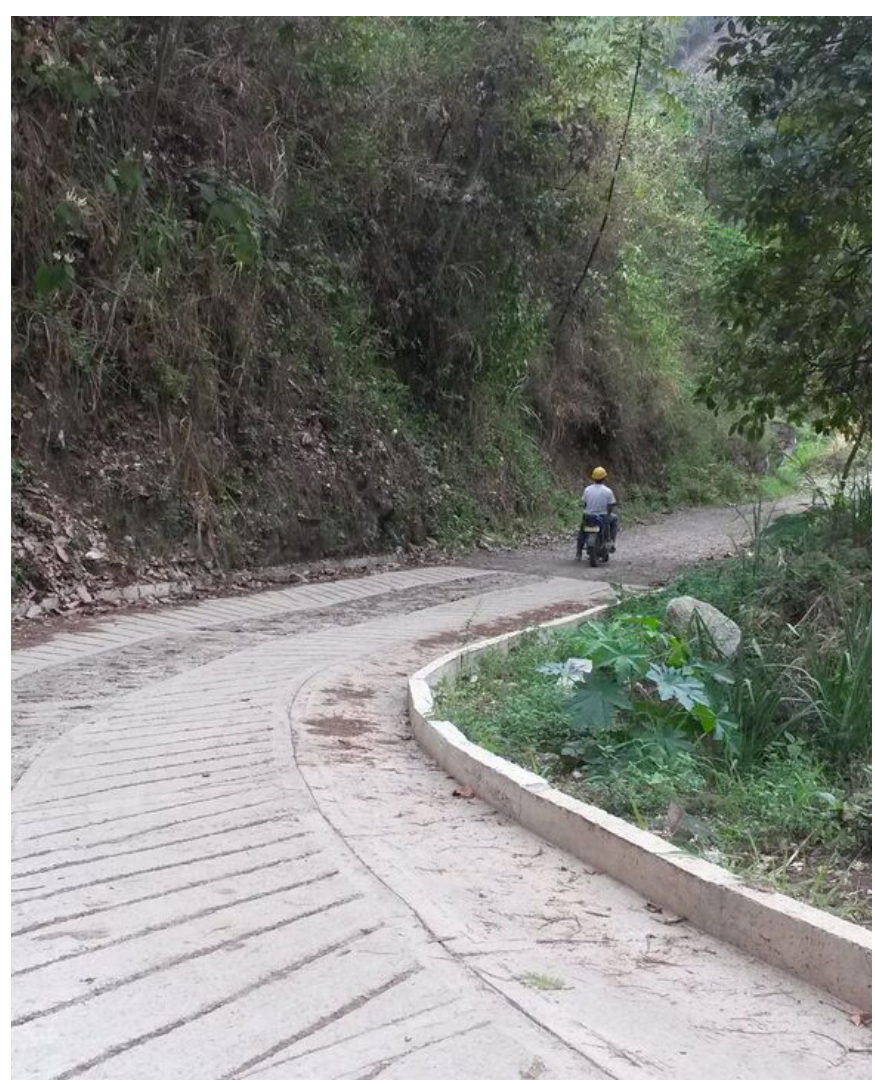

Vias terciarias colombianas. Fuente: TRANVIAS - SAS.

vez, deberían replicar el ejercicio y ceder el control de las vías terciarias (incluidos los caminos vecinales) a los municipios. Para esta transferencia, la ley creó el Fondo de Cofinanciación de Vías, por medio del cual se entregaría a los departamentos o municipios una suma fija para el mantenimiento de las vías asignadas. Pese al esfuerzo realizado, pocas vías fueron transferidas y siguen bajo la gestión nacional.

Consciente de las debilidades existentes en la gestión de la malla vial Secundaria, en el año 2008 el Gobierno Nacional tomó la iniciativa de adelantar el Plan Vial Regional (PVR)[4], con el objeto de apoyar a los departamentos para enfrentar su debilidad financiera e institucional y la ausencia de políticas de sostenibilidad y buen uso de las vías. Dicho programa incluía en cada departamento la realización de un inventario de longitudes, especificaciones geométricas y estado de las superficies viales, el cual debería servir de base para la elaboración de un Plan Vial Departamental, de acuerdo con criterios y lineamientos establecidos por el Ministerio de Transporte. Pese a que en los distintos 
departamentos se realizaron los inventarios viales, se identificaron proyectos y se definieron prioridades de inversión, los inventarios no han sido actualizados y muchos proyectos están sin ejecutar. Unos pocos han sido incluidos en programas de Contrato-Plan donde se busca unir esfuerzos entre la Nación y los departamentos en la ejecución de obras de pavimentación, rehabilitación y construcción de nuevas vías[5].

Según el inventario realizado por el PVR, la Red Vial Departamental tiene una longitud de $44.399 \mathrm{~km}$ de los cuales el 48,2\% corresponde a vías en afirmado; $24 \%$ a vías pavimentadas, y $28 \%$ a vías destapadas (en tierra). Solo el $31 \%$ de las vías pavimentadas se encuentran en buen estado.

Y por último está la Red Terciaria, que cumple una función de interconexiónentre las veredas, las cabeceras municipales y las carreteras departamentales. Muchas de estas vías son angostas y tienen fuertes pendientes, y solo cerca de $1.400 \mathrm{~km}$ están pavimentados. En esta red los volúmenes de tránsito son, en promedio, inferiores a 30 vehículos por día.

Esta malla vial se encuentra bajo la gestión de INVIAS, los departamentos y los municipios, y tiene una longitud de $27.577 \mathrm{~km}$, que representa el 18\% del total y corresponde a la red no transferida del antiguo Fondo Nacional de Caminos Vecinales. La red a cargo de los departamentos suma $13.959 \mathrm{~km}$ lo que equivale al $9 \%$, mientras que la red a cargo de los municipios alcanza los 100.419 km y representa el $65 \%$ del total. Existe también una red privada de caminos que ha sido construida para fines específicos, como pueden ser el acceso a proyectos de exploración y explotación petrolera. Esta red privada de caminos se estima en $12.251 \mathrm{~km}$, o sea el 8\%. Estas cifras no incluyen los caminos construidos ilegalmente por grupos al margen de la ley ni tampoco tampoco aquellos que soportan las actividades de la minería ilegal.

En la conformación de esta malla vial han participado distintas entidades gubernamentales, empresas privadas, e incluso los ciudadanos, estos últimos en forma individual o bajo diferentes modalidades de organización ciudadana, como las Juntas de Acción Comunal. Los pequeños caminos son las obras que verdaderamente llegan al campesino, apoyan la producción y comercialización de sus productos y facilitan su acceso a los servicios básicos, pero muchas de estas son simples trochas.

Claramente existe una diferencia notable entre aquellas áreas con y sin acceso vial. No en vano, distintos estudios destacan una relación directa entre pobreza, ruralidad y aislamiento geográfico"[6]. Los caminos incluidos en esta red son los que llegan a los más lejanos extremos de la geografía nacional, en apoyo de los campesinos y comunidades aisladas.

Hasta 1960 la gestión de los caminos vecinales a nivel

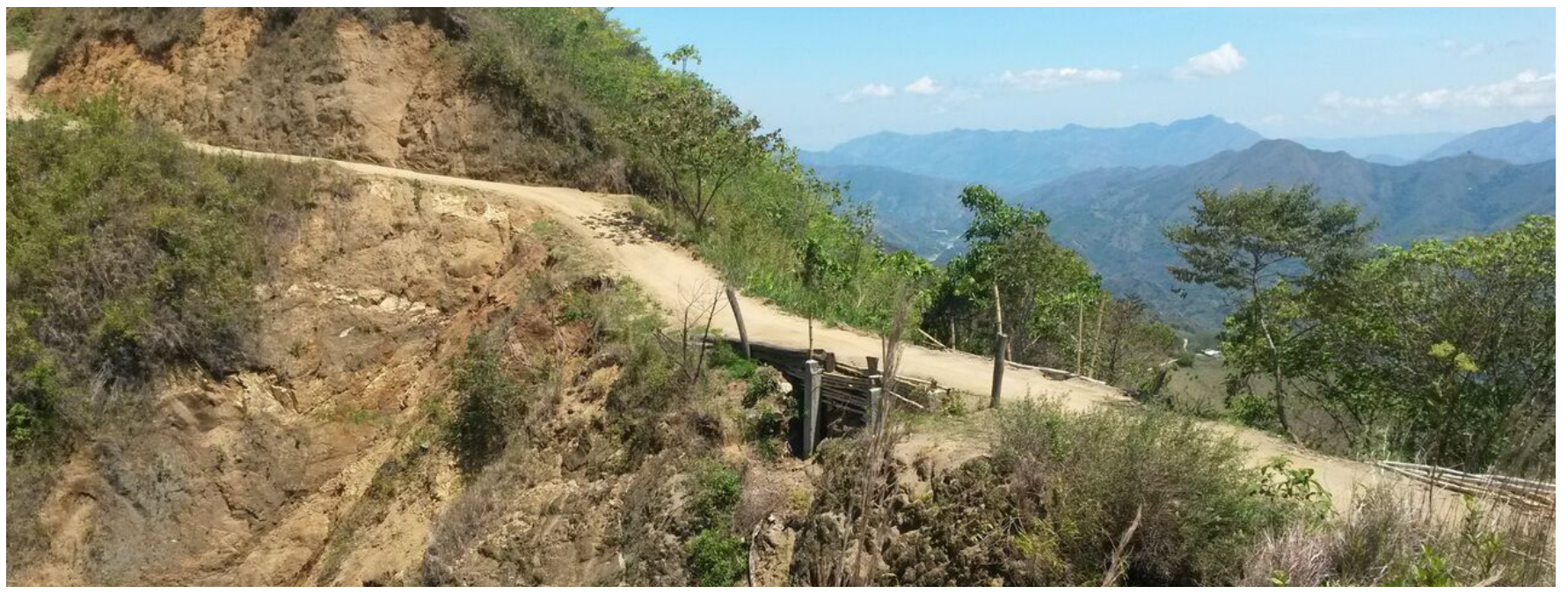

Vias terciarias colombianas. Fuente: TRANVIAS - SAS. 
nacional estuvo orientada por el Ministerio de Obras Públicas, y a nivel regional por los departamentos y municipios. En todos los casos, sin mayor coordinación o planificación, aunque siempre hubo conciencia de la importancia de este tipo de vías, ya fuese para colonización, acceso a comunidades en áreas aisladas, o para comunicación intra e intermunicipal. También siempre se destacaba su importancia en el proceso de recuperación de zonas que habían sido afectadas por la violencia, imperante en esos años[7].

Desde su creación, en 1960, el FNCV[8] tuvo como objetivo atender y coordinar la construcción, conservación y el mejoramiento de caminos regionales y vías locales, logrando grandes avances en la conformación de la malla vial terciaria. Aunque se utilizaban manuales y especificaciones de diseño, estos no estaban adecuados a las condiciones especiales de las vías con bajos volúmenes de tránsito.

El decreto extraordinario 77 de 1987, consignado en la Ley 12 de 1986, estableció la transferencia de recursos del IVA a los municipios, y obligó al FNCV a disminuir su tamaño y ejecutar solamente obras que fueran cofinanciadas por los municipios. En 1993, la Ley 105 fijó una política de descentralización y cofinanciamiento vial y se inició el proceso de desmonte del FNCV, aunque este fondo siguió operando hasta el año 2003.

\section{Gestión del FNCV}

1) Gestión de créditos con la banca multilateral para la construcción, mejoramiento y mantenimiento de caminos en todo el país.

2) Desarrollo de caminos en zonas de "colonización dirigida" con el INCORA y en programas DRI en diferentes áreas del país.

3) Con la Federación Nacional de Cafeteros fue notable la colaboración en zonas cafeteras, donde se llegó, incluso, hasta la pavimentación de algunos caminos.

4) Creó el programa Pico y Pala, mediante el cual se vinculaba a las comunidades en la construcción de sus propias vías.
Cuando se decretó su supresión, la Red terciaria a cargo del FNCV llegaba a más de 32.000 km, una longitud superior a la correspondiente a la red primaria a cargo del Ministerio de Obras Públicas. En cumplimiento de la Ley 105 del 93 se trató de transferir las vías que la conformaban a los municipios, pero esto solo se pudo realizar parcialmente[9] y las vías no transferidas pasaron a constituir lo que se conoce como la Red Terciaria, actualmente a cargo de INVIAS.

Por otra parte, con base en las solicitudes presentadas por los municipios para ejecutar recursos nacionales, INVIAS ha suscrito un gran número de convenios a través de los cuales se ha adquirido un mejor conocimiento sobre la conformación de la red terciaria. De acuerdo con INVIAS, entre los años 2010 y 2014 se suscribieron 2.626 convenios con 1034 municipios a través de los cuales se intervinieron 31.000 km de la red, incluyendo $1.300 \mathrm{~km}$ de placa - huella[10] y $90 \mathrm{~km}$ de pavimentación.

La conformación de la red terciaria no ha sido homogénea. Al igual que se comentó para el caso de las carreteras departamentales, los caminos vecinales y vías terciarias tienen diferentes tipos de especificaciones según la entidad ejecutora y en muy pocos casos se han tenido en cuenta consideraciones de cambio climático.

\section{Innovación y nuevas tecnologías}

Si bien es cierto que la red pavimentada en Colombia representa apenas el 11,3\%, no menos cierto es que la pavimentación de vías debe estar supeditada a los volúmenes de tránsito que tenga cada carretera, y no a presiones regionales o locales. En el caso de las vías de bajo tránsito[11], como son la mayor parte de las carreteras secundarias y terciarias del país, hay diversas opciones tecnológicas que permiten asegurar la transitabilidad de los caminos, manteniéndolos en buen estado, sin necesidad de recurrir a la pavimentación tradicional (concreto o asfalto).

El tema ha sido abordado en diferentes estudios nacionales e internacionales, ya que se trata de una problemática común en los países en vía de desarrollo. En Colombia se han dado algunos pasos para utilizar 
opciones alternas que aseguren la transitabilidad de los caminos mediante la estabilización de suelos con productos químicos. En este sentido, se han realizado ensayos en distintos tramos de prueba bajo la dirección del MinTransporte, INVIAS y algunas administraciones departamentales. Por ejemplo, en Antioquia se están analizando distintos productos en cinco tramos de prueba, en un programa coordinado por la Secretaría de Infraestructura que cuenta con la participación de las universidades de Antioquia, de Medellín y la Red Ennoviar.

Por otra parte, ya hay una experiencia interesante en el país con la utilización de placa huellas en diferentes regiones. De acuerdo con datos de INVIAS, actualmente existen más de 1.300 km, y se tiene prevista la construcción de 500 km más en unos pocos meses. Pero lo importante es poder aplicar tecnologías autóctonas a problemas autóctonos, en lo que tiene que ver con la disponibilidad de materiales locales y productos para mejorar su resistencia.

Adicionalmente es importante analizar las consecuencias del cambio climático en los caminos y la adaptación de tecnologías o especificaciones locales para minimizar este impacto.

\section{Inversiones en las redes secundaria y terciaria}

Los recursos para las redes secundaria y terciaria provienen de MinTransporte, a través del INVIAS, los presupuestos departamentales, los presupuestos municipales y los recursos del Sistema General de Regalías (SGR). En este sentido, cualquier ente territorial puede presentar ante los Órganos Colegiados de Administración y Decisión Regional (OCAD) proyectos viales para su financiamiento. Los OCAD revisan la solicitud y toman una decisión sobre la viabilidad del proyecto y la entidad ejecutora del mismo con base en la información existente.

El monto invertido en las redes Secundaria y Terciaria es significativo. Por ejemplo, la atención de vías incluidas en el programa de los Contratos-Plan, es de \$2.223.758 millones de pesos entre los años 2013 y 2017. Los proyectos aprobados a través del Sistema General de Regalías entre los años 2012 a 2013, alcanzan $\$ 2.284 .999$ millones de pesos. A esto hay que agregar las inversiones que realizan los departamentos con sus propios recursos.

Las inversiones en la red Terciaria a través del MinTransporte - INVIAS, entre 2010 y 2015, Ilega a $\$ 2.948 .101$ millones de pesos. Los recursos aprobados del Sistema General de Regalías suman \$1.456.715 millones de pesos entre los años 2012 y 2015. A las cifras anteriores hay que agregar lo correspondiente a inversiones municipales.

\section{El papel de las vías en la atención de zonas aisladas y marginadas}

Las vías secundarias y terciarias, por llegar a las más alejadas y marginadas zonas del país, siempre han jugado un papel importante en todos los planes de rehabilitación social y económica que se han llevado a cabo en las zonas de conflicto y violencia en el país, como el "Plan Nacional de Rehabilitación"[12], formulado en 1983, "Vías para la Paz"[13] formulado en 2000, y más recientemente "Caminos para la Prosperidad".

Con el Plan Nacional de Rehabilitación se buscaba reforzar la presencia del Estado en áreas marginadas y, simultáneamente, crear espacios de interlocución con las comunidades para superar las "condiciones objetivas" generadoras de violencia. El plan contempló la atención de 404 municipios con cerca de seis millones de habitantes, y también la atención de carreteras Primarias tales como la Troncal del Magdalena Medio, Santa Fe de Antioquia-Chigorodo, Chigorodó-Turbo y Villavicencio-San José del Guaviare, entre otras.

Con el plan de Vías para la Paz, la inversión en caminos hacía parte de una estrategia de inversión que incluía diversos programas[14] y se desarrollaba en zonas priorizadas por el Plan Colombia, específicamente en los municipios más pobres. Las áreas identificadas fueron el Magdalena Medio, el Sur Oriente, el Sur Occidente y otros. A nivel de vías terciarias se suscribieron convenios con los municipios para adelantar el mantenimiento y mejoramiento de esta red.

Algo similar sucede con el tercer programa aquí 


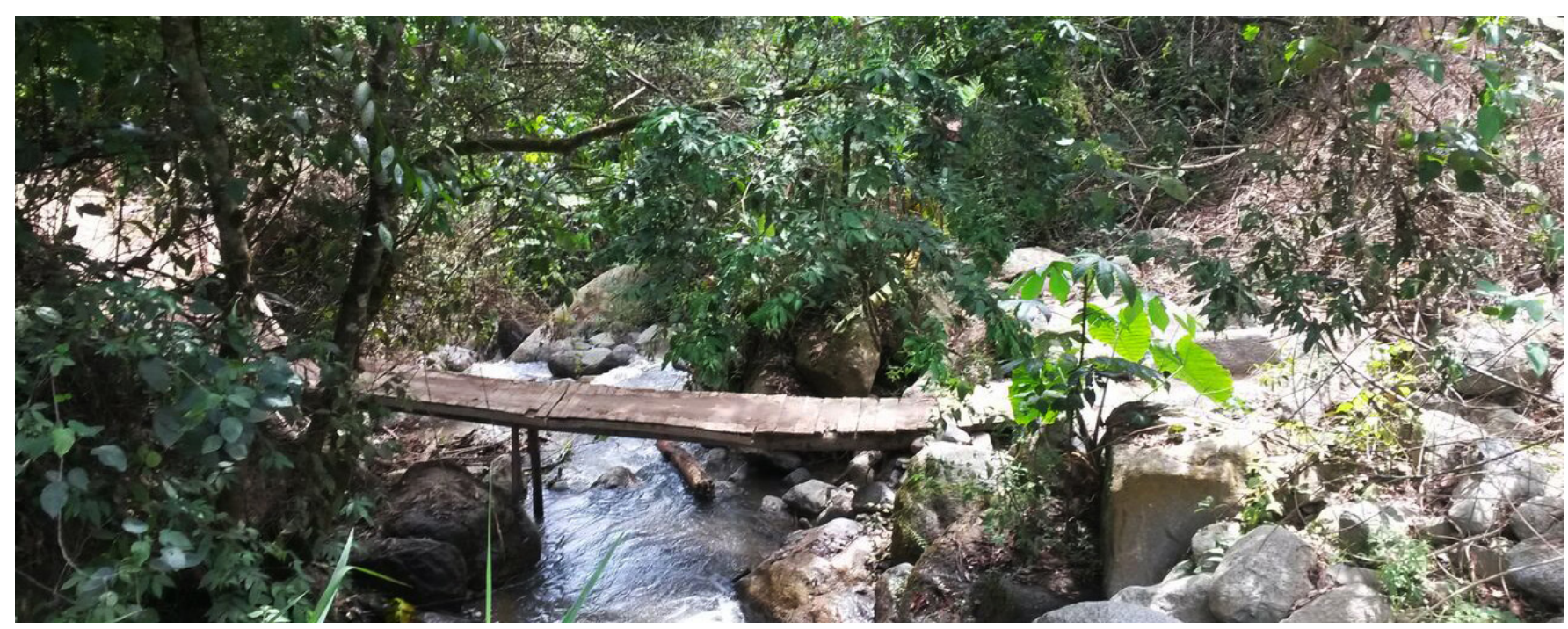

Vias terciarias colombianas. Fuente: TRANVIAS - SAS

mencionado, donde se han atendido carreteras Secundarias y Terciarias. En estas últimas se han suscrito más de 2.600 convenios con 1.034 municipios. Con esto ha sido posible mejorar el conocimiento sobre las vías en estos municipios, pero la información es aún insuficiente. Sin embargo la experiencia adquirida en este proceso es básica tanto para la elaboración de los inventarios viales municipales como para la preparación de proyectos viales con financiación de recursos de regalías y otros.

\section{Elementos para tener en cuenta en un plan de vías secundarias y terciarias}

\section{Institucionalidad y capacidad técnica}

Salvo unos pocos departamentos, la mayoría de los entes territoriales a nivel departamental, distrital y municipal se caracterizan por una debilidad institucional acentuada. En los departamentos se ha avanzado con el Plan Vial Regional, pero en los municipios falta mucho por hacer.

Aunque ya hay algunos manuales es necesario revisar lo existenteenmateriadenormas de diseñoyespecificaciones para adaptar este material a las condiciones locales, teniendo en cuenta el cambio climático.
Hasta ahora la Subdirección de Red Terciaria de INVIAS coordina y presta apoyo, pero hacia el futuro habría que pensar que si la Red Primaria queda totalmente bajo la gestión de la ANI, el INVIAS en su conjunto, debería evolucionar hacia una entidad de promoción y apoyo de las redes Secundaria y Terciaria.

\section{Planeación y preparación de proyectos}

A nivel departamental con el PVR se han realizado esfuerzos importantes. En los municipios esta actividad es incipiente. No hay coordinación con otros municipios ni tampoco con los departamentos. La mayoría de los caminos son obras intramunicipales, pero no se definen corredores de integración.

En las labores de planificación es importante contar con una visión de largo plazo más allá del periodo de los gobernadores y alcaldes. Con el PVR se cuenta con inventarios y planes, pero esta información no ha sido actualizada. En los municipios apenas se cuenta con información preliminar y se carece de inventarios de vías, no solo para planificar la red, sino también para facilitar el acceso a fuentes de inversión, como el SGR, por ejemplo. A lo anterior se suma el problema de los caminos ilegales, cuyo inventario e impacto deberá analizarse, ya sea para incorporarlos a la malla vial o para clausurar su utilización. 
El desconocimiento de la red no es solo físico sino también de su utilización. Cuántos y qué tipo de vehículos se movilizan por las carreteras son elementos desconocidos. Igual sucede en materia de costos de transporte.

En la preparación de los proyectos hay que considerar la posibilidad de avanzar por etapas. Especial atención merecen la realización de estudios y la adquisición de predios antes de iniciar la ejecución de las obras.

Para el posconflicto hay que identificar aquellas áreas donde se debe concentrar la inversión no solo en vías sino también en otros componentes, tal como ya se hizo en programas anteriores. En este sentido, hoy se cuenta mayor información sobre los municipios, lo cual permite una mejor selección de las áreas y las inversiones requeridas.

\section{Sostenibilidad de los activos viales}

Tradicionalmente se ha concedido prioridad a la ampliación nueva en longitud, en detrimento del mejoramiento y mantenimiento de lo existente. Tampoco se concede prioridad a mantener las vías en buen estado. Como resultado de esta situación, un alto porcentaje de las vías se encuentra en mal estado.

En las actividades de sostenibilidad es importante propiciar la participación de la comunidad, no solo para generar empleo rural, sino también para fortalecer el sentido de pertenencia sobre las vías. En este sentido existen diferentes opciones de participación que han sido utilizadas en diferentes programas en el país. Tal es el caso de las modalidades de peón caminero, convites y mingas, cooperativas de trabajo, microempresas de Pico y Pala, entre otras.

\section{Esquema contractual}

Se requiere transparencia en los procesos de las entidades y autorregulación de los participantes en las licitaciones. La transparencia en los procesos es muy importante. En muchas ocasiones solo hay un proponente, lo cual nunca es conveniente, tal como lo han señalado la Cámara Colombiana de la Infraestructura y otras entidades.

\section{Innovación y nuevas tecnologías}

No todas las vías tienen que tener su superficie pavimentada. Hay otras posibilidades que deben investigarse para mantener la transitabilidad en buenas condiciones. Otras opciones como las placa-huella constituyen unas innovaciones sencillas pero muy interesantes. Lo importante es definir un modelo de gestión sostenible bajo un marco institucional óptimo con manuales, especificaciones y fuentes de financiación

\section{Financiamiento}

Es necesario mejorar la calidad de la inversión. Siempre habrá limitación de recursos pero también mecanismos para para asegurar su eficiente utilización. En materia de financiamiento hay que aunar esfuerzos con los municipios, departamentos, la Nación y el SGR para mejorar la red y concentrarse en vías de mayor impacto. Un buen ejemplo de esto es el programa de contratosplan, mencionado anteriormente.

\section{Conclusiones}

Así como en el Plan Nacional de Transporte Intermodal se identificaron los principales proyectos de carreteras, en la malla vial primaria y en los departamentos, a través del PVR, se ha avanzado en la formulación de planes regionales para las vías secundarias. Ahora es necesario que a nivel local se cuente con inventarios y planes para las vías terciarias.

Vincular las áreas aisladas y remotas a la vida nacional reviste la mayor prioridad y, para ello, contar con una malla vial articulada y en buen estado juega un papel importante. Facilitar el acceso de bienes y servicios a estas comunidades así como posibilitar el transporte y comercialización de su producción agrícola en los centros de consumo, solo puede lograrse a través de caminos y carreteras articuladas que permitan reducir los costos de transporte.

Una mejor accesibilidad no solo se logra mejorando caminos individuales al interior de un municipio sino identificando corredores de integración cuya utilización se proyecta más allá del territorio de cada municipio. Esto solo se logra a través de un mejor conocimiento de las 
vías existentes y por ello la realización de inventarios y planes viales municipales es prioritaria.

Para mejorar la accesibilidad también es necesario acometer y mantener las vías en buen estado, independientemente del tipo de superficie que se tenga. En este sentido el uso de materiales locales, combinado con nuevos productos que mejoran la estabilización de los suelos o de tecnologías sencillas, como es la utilización de placa-huellas, debe promoverse.

Con un mejor conocimiento del estado de las vías secundarias y terciarias y de los proyectos identificados, es posible canalizar la inversión hacia áreas geográficas específicas y proyectos prioritarios. Por ejemplo, zonas críticas de desarrollo durante el posconflicto.

En la ejecución de las obras deben manejarse criterios de transparencia en todos aquellos procesos contractuales que deban emprenderse, así como también la utilización de manuales de diseño y normas técnicas apropiadas para las vías con bajos volúmenes de tránsito, incluyendo criterios que minimicen el posible impacto del cambio climático.

\section{Referencias}

(1) Ley 1228 del 16 julio 2008

(2) Plan Maestro de Transporte Intermodal - Una Política de Estado para hacer de Colombia un país más competitivo.

(3) Parsons, Brickerhoff, Quade \&amp; Douglas "Plan para el Mejoramiento del Transporte Nacional", diciembre de 1961.

(4) Documento CONPES 3478 "Politica para el Mejoramiento de la Gestión Vial a través del Plan Vial Regional", 25 julio 2008

(5) Se busca aunar esfuerzos de acuerdo con lo definido sobre vías y transporte en el Plan Nacional de Desarrollo, los Planes deDesarrollo Departamentales y los Planes Viales Departamentales.

(6) Leonardo Villar y Juan Mauricio Ramírez, Fedesarrollo, Working Paper 61 "Infraestructura Regional y Pobreza", Bogotá 2014.
(7) En esos años se hizo mucho énfasis en la generación de empleo. Algo parecido sucedió también con la construcción del ferrocarril del Atlántico

(8) Ley 1650 del 14 julio de 1960

(9) De $32.000 \mathrm{~km}$ so lo se transfirieron $6.000 \mathrm{~km}$ y quedaron aproximadamente 27.000 km por transferir los cuales siguen estando bajo la gestión de INVIAS.

(10) Las placa -huellas son placas en concreto reforzado que se colocan en caminos separadas por piedras fijadas en concreto.

(11) Corporación Andina de Fomento (CAF) "Soluciones e Innovaciones Tecnológicas de Mejoramiento de Vías de Bajo Transito, 2010

(12) Documento CONPES 2523 de 1991 "Plan Nacional de Rehabilitación 1991 - 1994: Orientación, Cobertura y Financiación"

(13) Documento CONPES 3075 de 2000 "Plan Colombia, Red de Apoyo Social: Programa Manos a la Obra. Componentes: Proyectos Comunitarios y Vías para la Paz"

(14) Empleos en Acción, Familias en Acción, Jóvenes en Acción, Vías para Paz, El campo en Acción, Atención Humanitaria, Derecho Internacional Humanitario. 